\title{
THE RADON-NIKODYM PROPERTY AND DENT ABLE SETS IN BANACH SPACES
}

\author{
W. J. DAVIS AND R。R.PHELPS ${ }^{1}$
}

ABSTRACT. In order to prove a Radon-Nikodym theorem for the Bochner integral, Rieffel [5] introduced the class of "dentable" subsets of Banach spaces. Maynard [3] later introduced the strictly larger class of " $s$-dentable" sets, and extended Rieffel's result to show that a Banach space has the Radon-Nikodym property if and only if every bounded nonempty subset of $E$ is $s$-dentable. He left open, however, the question as to whether, in a space with the Radon-Nikodym property, every bounded nonempry set is dentable. In the present note we give an elementary construction which shows this question has an affirmative answer.

Definitions. A Banach space $E$ has the Radon-Nikodym property if for each totally finite positive measure space $(X, \Sigma, \mu)$ and each $E$-valued, $\mu$ continuous measure $m$ on $\Sigma$ with $|m|(X)<\infty$, there is a Bochner integrable $f$ from $X$ to $E$ such that $m(E)=\int_{E} f d \mu(E \in \Sigma)$.

A subset $A$ of $E$ is dentable if for every $\epsilon>0$, there exists $x \in A$ such that $x \notin \operatorname{clco}\left(A \backslash S_{\epsilon}(x)\right)$. [Here $\operatorname{co} B$ denotes the convex hull of $B, \operatorname{cl} \operatorname{co} B$ is its closure and $S_{\epsilon}(x)=\{y \in E:\|x-y\| \leq \epsilon\}$.] A bounded set $A$ is called sdentable if for each $\epsilon>0$ there exists $x \in A$ such that $x \notin s\left(A \backslash S_{\epsilon}(x)\right)$. [Here $s(B)=\left\{\Sigma_{i=1}^{\infty} \lambda_{i} x_{i}: \lambda_{i} \geq 0, \Sigma \lambda_{i}=1,\left\{x_{i}\right\} \subset B\right\}$.] A point $x \in A$ is a denting [s. denting] point if for all $\epsilon>0, x \notin \mathrm{clco}\left(A \backslash S_{\epsilon}(x)\right)\left[x \notin s\left(A \backslash S_{\epsilon}(x)\right)\right]$.

Dentable sets are $s$-dentable, and Maynard has given an example of a bounded set which is $s$-dentable but not dentable. Rieffel has shown that if $A$ is not dentable, then neither is clco $A$. The analogous assertion fails for $s$-dentability: The closed unit ball of $C([0,1])$ is not dentable [5], but the constantly 1 function is an $s$-denting point. By Lemma 2 below, its interior is not $s$ dentable.

Lemma 1. A subset $A$ of $E$ is not dentable if and only if there exists

Received by the editors July 31, 1973.

AMS (MOS) subject classifications (1970). Primary 46G05; Secondary 28A45.

Key words and phrases. Banach space, dentable sets, vector valued measures, Radon-Nikodym theorem.

1 Research supported by the National Science Foundation Grant GP-28973. 
$\epsilon>0$ such that $A \subset \mathrm{cl} \operatorname{co}\left(A \backslash S_{\epsilon}(x)\right)$ for each $x \in A$. If $A$ is closed and convex, this is equivalent to $A=\operatorname{cl} \operatorname{co}\left(A \backslash S_{\epsilon}(x)\right)$ for each $x \in A$.

Proof. One implication is trivial. For the other, suppose $A$ is not dentable. Then there exists $2 \epsilon>0$ such that for each $y \in \dot{A}, y \in \operatorname{cl} \operatorname{co}\left(A \backslash S_{2 \epsilon}(y)\right)$. Suppose that $x, y \in A$ and $\|x-y\|>\epsilon$. Then $y \in \operatorname{cl} \operatorname{co}\left(A \backslash S_{\epsilon}(x)\right)$. On the other hand, if $\|x-y\| \leq \epsilon$, then $S_{\epsilon}(x) \subset S_{2 \epsilon}(y)$ so that $y \epsilon \operatorname{cl} \operatorname{co}\left(A \backslash S_{2 \epsilon}(y)\right) \subset$ $\operatorname{cl} \operatorname{co}\left(A \backslash S_{\epsilon}(x)\right)$, completing the proof.

Lemma 2. Suppose $C$ is a closed convex set in $E$ with nonempty interior (denoted by int $C$ ) and suppose $C$ is not dentable. Then there exists $\epsilon>0$ such that for each $x \in C$, int $C \subset \operatorname{co}\left[\right.$ int $\left.C \backslash S_{\epsilon}(x)\right]$. In particular, int $C$ is not s-dentable.

Proof. By Lemma 1, there exists $\epsilon>0$ such that $C=\operatorname{clco}\left(C \backslash S_{\epsilon}(x)\right)$ for each $x \in C$. Let $J_{x}=C \backslash S_{\epsilon}(x)$ so that int $J_{x}=\operatorname{int} C \backslash S_{\epsilon}(x)$. For $\epsilon$ sufficiently small, int $J_{x} \neq \varnothing$ for each $x \in C$. Fix $x$ and let $J=J_{x}$. Then $C=$ $\mathrm{clco} J$ and we want to show that int $(\operatorname{clcoJ}) \subset \operatorname{co}($ int $J)$. Note that $J \subset$ $\operatorname{cl}($ int $J):$ If $y \in J$, then $y \in C$ and $\|y-x\|>\epsilon$. Let $z \in$ int $C$, so that $[z, x)$ $C$ int $C$ and there exists $u \in[z, x) \cap S_{\epsilon}(x)$. Therefore, $[u, y) \subset$ int $C$ so for some $v \in[u, y)$ we have $[v, y) \subset \operatorname{int} C \backslash S_{\epsilon}(x)$. Thus $y \in \mathrm{cl}($ int $J)$. It now follows that $\operatorname{co} J \subset \mathrm{cl} \operatorname{co}($ int $J$ ). Taking the interior of each side, we conclude that

$$
\operatorname{int}(\mathrm{cl} \operatorname{co} J)=\operatorname{int}(\operatorname{co} J) \subset \operatorname{int}(\mathrm{cl} \operatorname{co}(\text { int } J))=\operatorname{co}(\text { int } J) \text {. }
$$

The equalities follow from the fact that the interior of a convex set coincides with the interior of its closure.

Proposition. If $E$ contains a bounded nonempty set which is not dent able, then it contains a bounded closed convex and symmetric set $C$ which is not dentable and which has nonempty interior. In particular, $E$ can be renormed so that the new unit ball is not dentable and the interior of the new unit ball is not s-dentable.

Proof. If $A$ is a bounded nonempty set which is not dentable, then the same is true of the sets $A_{1}=A \cup(-A)$ (definition), $A_{2}=\mathrm{cl} \operatorname{co} A_{1}$ (Rieffel [5, Proposition 2]) and $A_{3}=S+A_{2}$, where $S$ is the closed unit ball of $E$ (easy computation). Let $C$ be the closure of $A_{3}$. Again, by Rieffel's proposition, $C$ is not dentable. By Lemma 2 , int $C$ is not $s$-dentable.

What we have shown is that every bounded subset of $E$ is dentable if and only if every bounded subset of $E$ is $s$-dentable. This yields the following corollary. 
Corollary: A Banach space $E$ has the Radon-Nikodym property if and only if every bounded nonempty set in $E$ is dentable.

J. Diestel has raised the question of the relationship between the RadonNikodym property and the Krein-Milman property [every closed bounded convex set is the closed convex hull of its extreme points]. It is known that a space has both properties if it is reflexive or is a separable conjugate space [1], [4]. The Proposition shows that every bounded nonempty subset of $E$ is dentable if and only if the unit ball of every Banach space isomorphic to $E$ is dentable. Is the analogous assertion true for the KreinMilman property? That is, if $E$ contains a closed, bounded nonempty convex set which is not the closed convex hull of its extreme points, can $E$ be renormed so that its new unit ball is not the closed convex hull of its extreme points? The answer is affirmative for the spaces $(m)$ and $L_{1}([0,1])[2]$. In spaces with the Radon-Nikodym property does every closed bounded convex set have a denting point ( $s$-denting point, extreme point)?

Added in proof. Since this paper was submitted, a number of related results have appeared. Huff [6] has given an independent proof of the above corollary, by modifying the proof of Maynard's main theorem [3]. Lindenstrauss has used the corollary to show (cf. [7]) that the Radon-Nikodym property implies the Krein-Milman property, and the question conceming denting points has also received an affirmative answer in [7]. Huff and Morris [8] have shown that the Krein-Milman property implies the RadonNikodym property in conjugate spaces, but this implication remains open for arbitrary spaces. Finally, Edgar [9] has generalized Lindenstrauss' result by obtaining a Choquet-type representation theorem for bounded closed convex subsets of a Banach space with the Radon-Nikodym property.

\section{BIBLIOGRAPHY}

1. C. Bessaga and A. Pelczyński, On extreme points in separable conjugate spaces, Israel J. Math. 4 (1966), 262-264. MR 35 \#2126.

2. V. L. Klee, Some characterizations of reflexivity, Rev。Ci。Lima 52 (1950), 15-23. MR 13, 250。

3. H. B. Maynard, A geometrical characterization of Banach spaces having the Radon-Nikodým property, Trans. Amer. Math. Soc. 185 (1973), 493-500.

4. I. Namioka, Neighborhoods of extreme points, Israel J. Math. 5 (1967), 145152. MR $36 \# 4323$.

5. M. A. Rieffel, Dentable subsets of Banach spaces, with application to a Radon-Nikodým theorem, Functional Analysis (Proc. Conf., Irvine, Calif., 1966), Academic Press, London; Thompson Book Co., Washington, D. C., 1967, pp. 71-77. MR $36 \# 5668$. 
6. R.E.Huff, Dentability and the Radon-Nikodym property, Duke Math。J. (to appear).

7. R. R. Phelps, Dentability and extreme points in Banach spaces, J. Functional Analysis (to appear).

8. R. E. Huff and P. D. Morris, Dual spaces with the Krein-Milman property have the Radon-Nikodým property, Proc。Amer. Math. Soc。(to appear)。

9. G. A. Edgar, A noncompact Choquet theorem.

DEPARTMENT OF MATHEMATICS, OHIO STATE UNIVERSITY, COLUMBUS, OHIO 43210

DEPARTMENT OF MATHEMATICS, UNIVERSITY OF WASHINGTON, SEATTLE, WASH• INGTON 98195 